\title{
PER UN'EDIZIONE DELLA VERSIONE 'JOHANNES' DELL'HISTORIA KAROLI MAGNI ET ROTHOLANDI
}

\author{
Laura RAMELLO \\ laura.ramello@unito.it \\ Università di Torino
}

\begin{abstract}
Le prime traduzioni volgari dell'Historia Karoli Magni et Rotholandi $i^{1}$ - o Cronaca dello Pseudo Turpino - iniziarono a comparire in terra francese a mezzo secolo di distanza dalla stesura del testo latino; la complessità della loro tradizione, in cui non mancano incroci e contaminazioni, concorre a delineare un panorama assai nebuloso, che la bibliografia esistente contribuisce solo in parte a definire.

L'edizione della versione provenzale ${ }^{2}$, ha rappresentato il primo tassello di un complessivo intervento di messa a punto, sul piano filologico, dei testi di tradizione gallo-romanza, nonché dell'individuazione dei contesti storico-culturali in cui essi furono elaborati ${ }^{3}$; l'attuazione di questa prassi si rende ora indispensabile per le traduzioni di ambito oitanico in quanto, nonostante le indagini pregresse, il loro quadro appare solo in parte chiarito.
\end{abstract}

\footnotetext{
${ }^{1}$ Per un excursus sulla fortuna del testo in Europa cfr. Jean-Claude Vallecalle, «La réception de la Chronique du Pseudo-Turpin en Europe», Cahiers de recherches médiévales et humanistes, 25 (2013), pp. 465-469. [Online]. Link: <http://crm.revues.org/13122> [Consultato il 14/12/2017]; Marianne Ailes, Suzanne Leedham, «Le Pseudo-Turpin en Angleterre», Cahiers de recherches médiévales et humanistes, 25 (2013), pp. 495-517. [Online]. Link: $<$ http://crm.revues.org/13127> [Consultato il 15/12/2017]. DOI: 10.4000/crm.13127; Danielle Buschinger, «La réception du Pseudo-Turpin en Allemagne au Moyen Âge», Cahiers de recherches médiévales et humanistes, 25 (2013), pp. 519-530. [Online]. Link: http://crm.revues. org/13130. DOI: 10.4000/crm.13130; Santiago López Martínez-Morás, «Le Pseudo-Turpin en Espagne», Cahiers de recherches médiévales et humanistes, 25 (2013), pp. 471-494. [Online]. Link: <http://crm.revues.org/13124>. [Consultato il 20/12/2017]. DOI: 10.4000/crm.13124. Per i più recenti sviluppi delle ricerche sul tema si veda ora Marco Piccat e Laura Ramello (dir.), L'Historia Turpini in Europa: ricerche e prospettive, Alessandria, Edizioni dell'Orso, 2019. Una traduzione in inglese moderno dell'Historia è reperibile in Kevin R. Poole, The Chronicle of Pseudo-Turpin, New York, Italica Press, 2004.

${ }^{2}$ Marco Piccat, Lo Pseudo-Turpino in antico provenzale. Edizione con introduzione e note critiche, Tübingen, Niemeyer, 2001.

${ }^{3}$ Sulle questioni inerenti la committenza dei volgarizzamenti, anche turpiniani, cfr. Gabrielle M. Spiegel, Romancing the Past. The Rise of Vernacular Prose Historiography in Thirteenth-Century France, Berkeley-Los Angeles-Oxford, University of California Press, 1993; Frédéric Duval, «Quel passé pour quel Moyen Âge?», in C. Galderisi (dir.), Translations médiévales. Cinq siècles de traductions en français au Moyen Âge (XI $I^{e} X V^{e}$ siècles), Turnhout, Brepols, 2011, vol. I, pp. 47-92; Sylvie Lefèvre, «Les acteurs de la traduction: commanditaires et destinataires. Milieux de production et de diffusion», in ibidem, pp. 147-206.
} 
I repertori bibliografici di prima consultazione forniscono dati diversi riguardo alla classificazione delle versioni, sia sul piano dell'individuazione del loro numero che dal punto di vista della successione cronologica; le limitate risorse elettroniche disponibili non aiutano affatto a dipanare i dubbi: pur tenendo conto di quanto riporta la home page del sito, che avverte della possibile incompletezza di alcune pagine, la scheda bibliografica della Chronique contenuta negli Archives de Littérature du Moyen Age (ARLIMA) ${ }^{4}$ risulta fortemente al di sotto di uno standard scientificamente accettabile, e ciò a causa della grave frammentarietà dei dati.

\section{LA VERSIONE 'JOHANNES’ NEL QUADRO DEI VOLGARIZZAMENTI OITANICI}

Per cogliere la complessità della questione è necessario richiamare brevemente le ipotesi tassonomiche elaborate sino ad ora: nel suo articolo per il Grundriss der Romanischen Literaturen des Mittelalters, Jacques Horrent ${ }^{5}$ parla di versioni pittavine e anglonormanne, fissandone il numero a sette, senza ulteriori precisazioni; nel Dictionnaire des lettres françaises (DLFr), Gillette Tyl-Labory ${ }^{6}$ richiama l'esistenza di almeno sei traduzioni, cronologicamente distribuite secondo la seguente tabella:

\begin{tabular}{|l|l|}
\hline Successione cronologica & Sequenza nel DLFr \\
\hline $\begin{array}{l}\text { A. Traduzione di Nicolas de Senlis (1195-1205) } \\
\text { (in Chronique Saintongeaise, 1205-1230); }\end{array}$ & I. = D (detta Turpin I) \\
$\begin{array}{l}\text { B. Traduzione 'Johannes' (1206-1207); } \\
\begin{array}{l}\text { C. Traduzione anglonormanna (Williame de } \quad \text { Briane, 1210-1220); } \\
\text { D. Traduzione anonima (1210-1220); }\end{array}\end{array}$ & III. = B (detta Turpin II) \\
$\begin{array}{l}\text { E. Traduzione anonima (ante 1240); } \\
\text { F. Traduzione borgognona (fine XIII sec.) }\end{array}$ & IV. $=$ C \\
\hline
\end{tabular}

Nella sequenza cronologica dei volgarizzamenti più antichi, la versione 'Johannes' si colloca al secondo posto dopo quella di Nicolas de Senlis (1195-1205), perduta nella sua stesura originaria, ma inglobata, con interpolazioni, all'interno della Chronique Saintongeaise (1205-1230).

${ }^{4}$ ARLIMA: Archives de Littérature du Moyen Age <www.arlima.net/mp/pseudo-turpin. html>.

${ }^{5}$ Jacques Horrent, «Pseudo-Turpin», in Rita Lejeune (dir.), Grundriss der Romanischen Literaturen des Mittelalters (Les épopées romanes), t. III/1, Heidelberg, C. Winter Universitätsverlag, 1981, pp. 30-34; III/2, Heidelberg, C. Winter Universitätsverlag, 1985, p. 103.

${ }^{6}$ Gillette Tyl-Labory, «Chronique du Pseudo-Turpin», in Robert Bossuat, Louis Pichard e Guy Raynaud de Lage (edd.), Dictionnaire des lettres françaises, Paris, Fayard, 1994², p. 294. 
Tuttavia, nella sua trattazione la Tyl-Labory ${ }^{7}$ stravolge questa cronologia, anticipando ai primi posti le versioni $\mathrm{D}$ e E, cui seguono B e $\mathrm{C}$ mentre $\mathrm{A}$, traduzione teoricamente più antica, scivola al quinto posto; la sua dislocazione, a priori non del tutto improponibile se si tiene conto non della datazione del perduto testo originario, bensì di quello che lo veicola, avviene tuttavia nell'ambito di una classificazione in cui il tentativo di recupero della corretta sequenza temporale si verifica solo nelle posizioni C-F, mentre ai primi posti vengono collocate due versioni che alterano tale ordine.

Le ragioni dell'incongruenza vanno probabilmente ricercate nella pregressa tradizione scientifica sull'argomento: nel 1880 Fredrik Wulff, fra i primi ad occuparsi dei volgarizzamenti turpiniani oitanici, pubblicò $\mathrm{D}$ e E identificandole come Chronique de Turpin I e Chronique de Turpin $I I^{8}$; le denominazioni furono mantenute da Ronald N. Walpole che, a distanza di un secolo, curò la riedizione dei testi ${ }^{9}$; pur rettificandosi in seguito ${ }^{10}$, in una fase della sua indagine egli attribuì a B l'identificativo Turpin III; nella classificazione del DLFr si legge dunque il tentativo di conciliare una tradizione speculativa più o meno cristallizzata con la cronologia reale.

Nel repertorio di Brian Woledge ${ }^{11}$, che adotta un criterio cronologico tout-court, si citano sette ${ }^{12}$ versioni; lo scarto di un'unità rispetto alla sequenza di cui sopra è dovuto al fatto che egli considera il testo su cui intervenne Pierre de Beauvais come indipendente da B; gli aggiornamenti apportati nel Supplemento al repertorio, che riflettono ampiamente le ipotesi di Walpole, modificano parzialmente quest'impostazione, accorpando in sostanza le due versioni ed individuando al loro interno una serie di sottotipologie. In coda al paragrafo dedicato a $\mathrm{F}$, Woledge $\mathrm{e}^{13}$ accenna nondimeno ad un'ottava versione, facente parte delle Grandes Chroniques de France; il quadro che ne risulta è il seguente:

${ }^{7}$ Ibid., p. 295.

${ }^{8}$ Fredrik Wulff, «La Chronique dite de Turpin, publiée d'après deux anciens textes français, I: texte contenu dans le ms. BN. fr. 1850; II: texte contenu dans le ms. fr. 2137», Acta Universitatis Lundensis. Lunds Universitets Ars-Skrift, 16 (1879-1880), t. I, pp. i-vi, 1-42; t. II, pp. 43-76.

${ }^{9}$ Ronald N. Walpole, An Anonymous old French Translation of the Pseudo-Turpin Chronicle: A Critical Edition of the Text Contained in Bibliothèque Nationale Mss fr. 2137 and 17203 and incorporated by Philippe Mouskés in his Chronique rimée, Cambridge, Massachusetts, The Mediaeval Academy of America, 1979; Ronald N. Walpole, Le Turpin français, dit le Turpin I, Toronto-Buffalo-London, University of Toronto Press, 1985.

${ }^{10}$ Ronald N. Walpole, Le Turpin français, op. cit., p. XV.

${ }^{11}$ Brian Woledge, Bibliographie des romans et nouvelles en prose française antérieurs à 1500, Genève, Droz, 1975, pp. 100-103.

${ }^{12}$ Brian Woledge, Bibliographie des romans et nouvelles en prose française antérieurs à 1500, Supplément, Genève, Droz, 1975, pp. 82-89.

${ }^{13}$ Brian Woledge, Bibliographie des romans, op. cit., p. 103. 


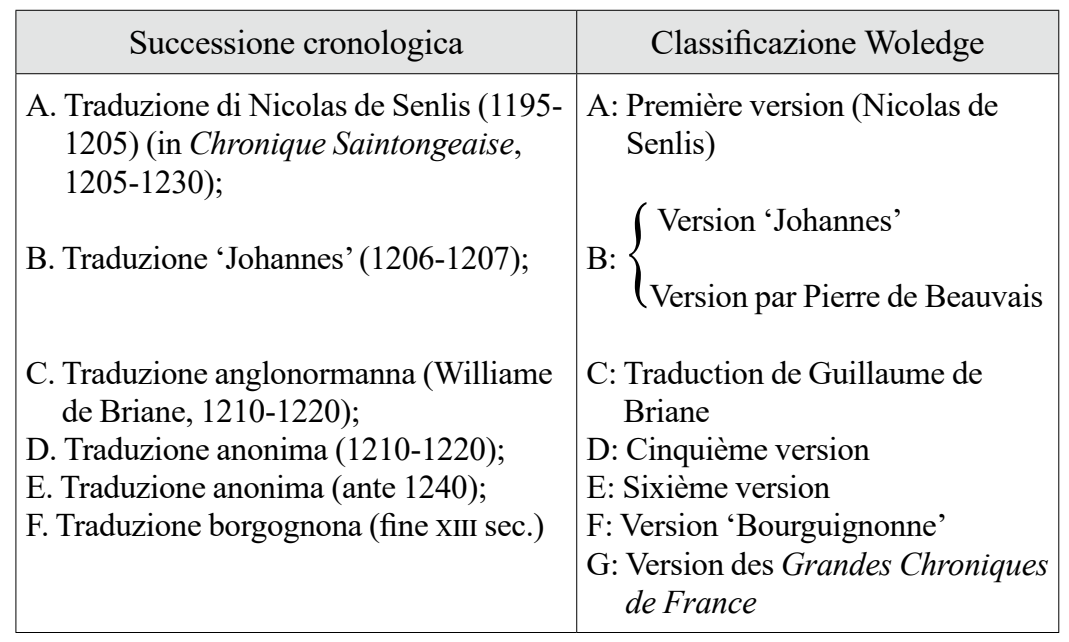

Nel 1973 Ian Short, editore della traduzione anglonormanna, ribadendo l'esistenza di almeno sei versioni indipendenti ante 1250 , ne identificò altre dieci di lì alla prima metà del XVI secolo; pur impostata su base cronologica, la sua classificazione ${ }^{14}$ coincide solo parzialmente con l'ordinamento prima richiamato:

\begin{tabular}{|c|c|}
\hline Successione cronologica & Classificazione Short \\
\hline $\begin{array}{l}\text { A. Traduzione di Nicolas de Senlis } \\
(1195-1205) \text { (in Chronique } \\
\text { Saintongeaise, 1205-1230); }\end{array}$ & - A. Traduzione di Nicolas de Senlis \\
\hline $\begin{array}{l}\text { B. Traduzione 'Johannes' } \\
\text { (1206-1207); }\end{array}$ & - B. Traduzione 'Johannes' \\
\hline $\begin{array}{l}\text { C. Traduzione anglonormanna } \\
\text { (Williame de Briane, } \\
\text { 1210-1220); }\end{array}$ & $\begin{array}{l}\text { - C. Traduzione anglonormanna } \\
\text { (William de Briane) }\end{array}$ \\
\hline $\begin{array}{l}\text { D. Traduzione anonima } \\
(1210-1220)\end{array}$ & -D. $\left\{\begin{array}{l}\text { Traduzione contenuta nella } \\
\text { Cronaca del ms. Vat. Regina } 624 \\
\text { Versione usata dall'Anonyme de } \\
\text { Béthune }\end{array}\right.$ \\
\hline E. Traduzione anonima (ante 1240); & $\begin{array}{l}\text { - E. Versione della Chronique Rimée di } \\
\text { Philippe Mouskés } \\
\text { - Traduzione del Ménestrel d'Alphonse } \\
\text { de Poitiers } \\
\text { - Versione di Primat (Grandes } \\
\text { Chroniques de France) }\end{array}$ \\
\hline $\begin{array}{l}\text { F. Traduzione borgognona (fine XIII } \\
\text { sec.) }\end{array}$ & $\begin{array}{l}\text { - F. Traduzione borgognona } \\
\text { - Traduzione del Rouergue }\end{array}$ \\
\hline
\end{tabular}

${ }^{14}$ Ian Short, The Anglo-Norman Pseudo-Turpin Chronicle of William of Briane, Oxford, Blackwell for the Anglo-Norman Text Society, 1973, p. 2, nota 4. 
- Versione di Jean de Vignay nello Speculum Historiale

- Versione di Jean de Mansel nel Fleur des Histoires (1450)

- Versione di Jean Baignon nel Roman de Fierabras (1478)

- Versione di Nicole Gilles negli Annales

- Versione del Miroir Historial, incunabolo (1495)

- Edizione a stampa del 1527

All'identità delle posizioni A, B, C, fa infatti seguito l'indicazione di due versioni (quella contenuta nella Cronaca tradita dal Ms. Vat. Regina 624 e quella usata dall'Anonyme de Béthune) corrispondenti a D ma ritenute indipendenti, il riferimento alla Chronique Rimée di Philippe Mouskés che riprende E, rimaneggiandola, e l'inserzione, nell'elenco, della Traduzione del Ménestrel di Alphonse de Poitiers e della versione contenuta nelle Grandes Chroniques de France di Primat, con il conseguente slittamento di $\mathrm{F}$ al nono posto.

La classificazione Short evidenzia una delle peculiarità della tradizione volgare dell'Historia Karoli Magni et Rotholandi, cioè il fatto che la Cronaca costituisca spesso parte integrante di compilazioni a carattere storico di più vasto respiro; il fatto rappresenta un problema a livello di valutazione di queste redazioni: esse vanno considerate alla stregua di versioni indipendenti e quindi da analizzare separatamente, o, nel caso in cui riprendano traduzioni precedenti, è legittimo, se non necessario, tenerne conto in fase di recensio e collatio codicum?

\section{LA TRADITIO CODICUM E LE EDIZIONI DELLA VERSIONE 'JOHANNES'}

Oltre a rappresentare una delle versioni più antiche, la 'Johannes' offre anche la tradizione più complessa: l'originario volgarizzamento dell'ipotetico 'Johannes' venne ripreso da Pierre de Beauvais, il quale gli antepose la sua traduzione della Descriptio qualiter Karolus Magnus clavum et coronam Domini a Constantinopoli Aquis Grani detulerit, racconto della leggendaria spedizione di Carlomagno a Costantinopoli; nel giustapporre i testi, Pierre de Beauvais intendeva offrire al pubblico, in una sequenza testuale organica, la narrazione dell'antefatto delle guerre di Carlo in Spagna raccontate dallo Pseudo-Turpino. A seguito di ciò, un anonimo rimaneggiatore effettuò un'ulteriore operazione di taglio-innesto, sintetizzando la Descriptio e incorporandola nel cap. I della Cronaca, intenzionalmente alimentando, in tal modo, l'impressione che lo Pseudo-Turpino fosse l'autore di entrambi i testi; la versione che ne scaturì godette di notevole 
successo, almeno a giudicare dalla nutrita tradizione manoscritta (ventitré codici) ad oggi conservata. All'interno di questo gruppo sono individuabili due sottogruppi, rappresentati rispettivamente da otto e quattordici testimoni ${ }^{15}$, sulla base dei nomi dei committenti (Renaud de Boulogne e Michel de Harnes) che compaiono nei prologhi.

Le analisi di Walpole rappresentano, nel bene e nel male, il punto di partenza obbligato per qualunque indagine sulle versioni oitaniche dello Pseudo Turpino; a lui si devono infatti le riedizioni delle due traduzioni anonime Turpin I e Turpin II e la pubblicazione del volgarizzamento borgognone ${ }^{16}$, che affiancarono l'edizione della versione di Nicolas de Senlis ripresa nella Chronique Saintongeaise curata da André de Mandach ${ }^{17}$ e la pubblicazione del volgarizzamento anglonormanno ad opera dello stesso De Mandach nel $1963^{18}$ e di Short dieci anni più tardi ${ }^{19}$.

A Walpole si deve soprattutto lo studio più approfondito della traditio della 'Johannes': egli censì trentadue codici, suddivisi in tre famiglie, le quali riflettono le tappe della vicenda evolutiva del testo:

1. Traduzione originale (4 mss.)

2. Versione di Pierre de Beauvais (5 mss.): traduzione della Descriptio + Cronaca dello Pseudo-Turpino

3. Descriptio riassunta e incorporata nel $1^{\circ}$ cap. dello PseudoTurpino (23 mss. $)^{20}$

Sottofamiglia a: Committente: Renaud de Boulogne ( 8 mss.) Sottofamiglia b: Committente: Michel de Harnes (14 mss.)

La disamina della tradizione fu propedeutica all'edizione del testo $^{21}$ ma, contrariamente a quanto il titolo - The Old French Johannes Translation of the Pseudo-Turpin Chronicle - dichiara, Walpole pubblicò non la traduzione originale, bensì il rimaneggiamento frutto della fase (3). L'editore peraltro non fornisce alcuna giustificazione

${ }^{15}$ Alla somma dei mss. delle sottofamiglie a e b va aggiunto il codice Bruxelles, KBR, 10233-36, che Walpole non riconduce a nessuno dei due sottogruppi.

${ }^{16}$ Ronald N. Walpole, «The Burgundian Translation of the Pseudo-Turpin Chronicle in Bibliothèque Nationale French Ms. 25438, part I», Romance Philology, 2 (1948-1949), pp. 177-215; «part II», Romance Philology, 3 (1949-1950), pp. 83-116.

${ }^{17}$ André de Mandach, Chronique dite Saintongeaise, texte franco-occitan inédit 'Lee'. À la découverte d'une chronique gasconne du XIII siècle et de sa poitevinisation, Tübingen, Niemeyer, 1970.

${ }^{18}$ André De Mandach, Chronique de Turpin, texte anglo-normand inédit de Willem de Briane (Arundel 220), in André de Mandach, Naissance et développement de la chanson de geste en Europe, vol. II, Genève-Paris, Droz, 1963.

${ }^{19}$ Ian Short, The Anglo-Norman Pseudo-Turpin..., op. cit.

${ }^{20} \mathrm{Cfr}$. nota 15 .

${ }^{21}$ Ronald N. Walpole, The Old French Johannes Translation of the Pseudo-Turpin Chronicle. A critical edition and supplement, Berkeley-Los Angeles-London, University of California Press, 1976. 


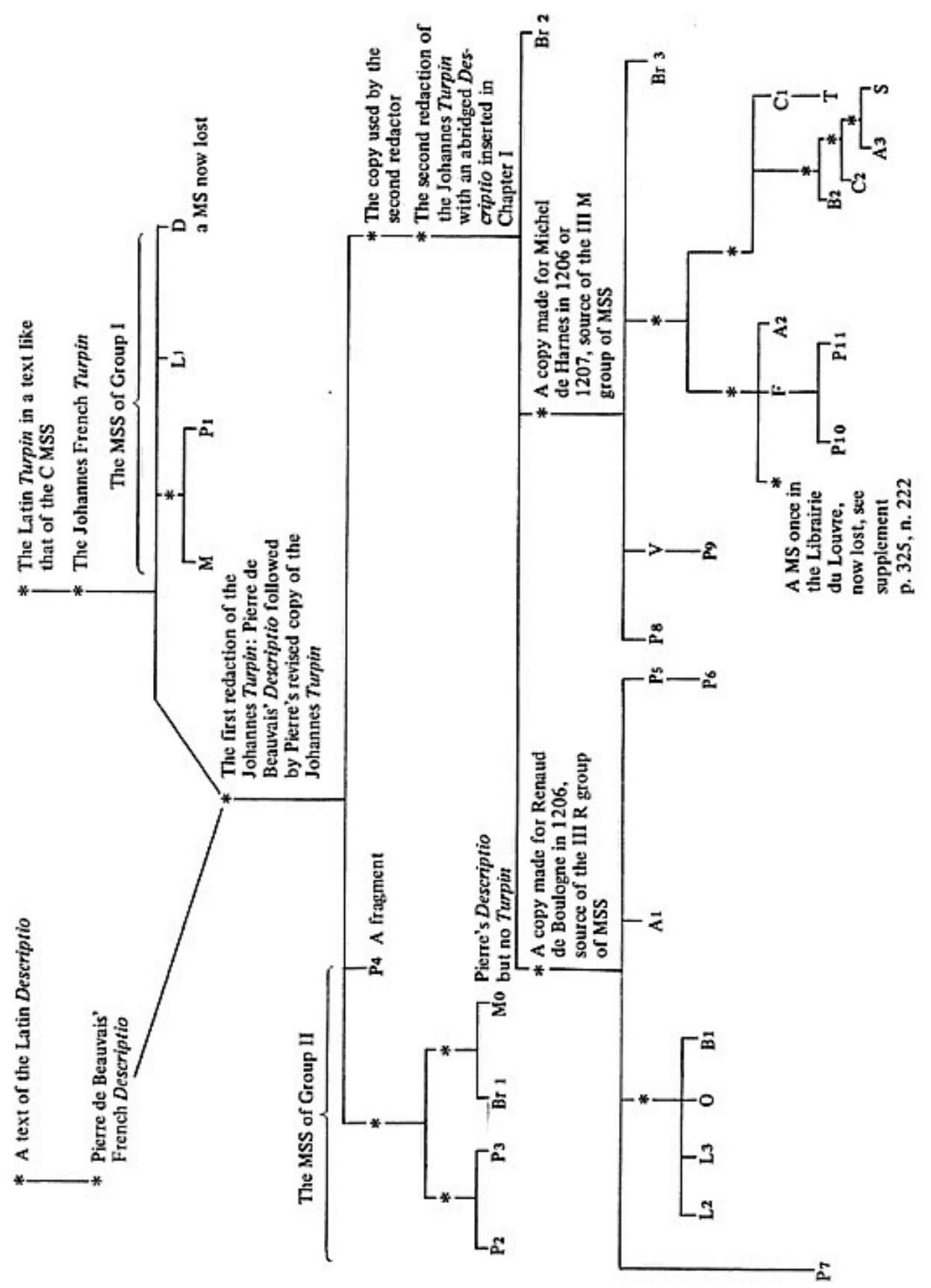


filologica a supporto della sua scelta, motivandola semplicemente sulla base della fortuna di questa versione nel Medioevo, e dunque sul piano della consistenza della tradizione manoscritta, opzione fors'anche legittima, ma in palese contrasto con quanto indicato nel frontespizio dell'edizione. Il testo edito da Walpole non è insomma la traduzione di 'Johannes'.

La paternità dell'opera appare ancora controversa; com'è noto, l'attribuzione si deve a Gaston Paris ${ }^{22}$ : sulla base del contenuto dell'explicit di un paio di codici, egli ipotizzò, sebbene con formula dubitativa, che un certo «Maistre Jehan» fosse stato l'artefice della traduzione («que maistre Jehan translata»); la questione venne ripresa e ampiamente dibattuta da Walpole ${ }^{23}$ sulla base di una serie di dati che Paris ignorava, ma che tuttavia non lo sottrassero alla stessa problematica conclusione: "we must leave open the possibility that a Maistres Jehans, known by tradition to him but lost to us, was indeed the author of our translation.... $\rangle^{24}$. Pur avendo sollevato una questione di assoluto rilievo, e cioè l'eventualità di un'attribuzione della versione a Pierre de Beauvais, sulla base delle non poche affinità stilistiche esistenti fra la Cronaca e la Descriptio - ipotesi già avanzata da Meyer e sulla quale Short ${ }^{25}$ si era dimostrato possibilista - nell'impraticabilità di condurre uno studio comparativo, dal punto di vista stilistico, esteso all'intero corpus testuale di Pierre, Walpole preferì rimanere ancorato alla tradizionale ipotesi di attribuzione a uno dei tanti 'Giovanni' ragionevolmente individuabili e continuare a credere che uno di essi potesse essere stato l'artefice della traduzione.

La tesi di Gabrielle M. Spiegel, che ritiene che il «Maistre Jehan» possa essere individuato in un «Johannes whose presence is recorded at the court of King John of England as the chaplain of Renaud of Dammartin, count of Boulogne ${ }^{26}$ è stata confutata da Marco Piccat sulla base di incongruenze cronologiche ${ }^{27}$; Piccat argomenta invece a favore di una possibile identificazione con Johannes de Barastre, maestro di teologia all'Università di Parigi, esponente dell'ordine domenicano - che vantava, come noto, forti legami con il culto e il pellegrinaggio jacopeo, a Parigi e non solo - e buon conoscitore della storia del viaggio di Carlomagno in Spagna ${ }^{28}$.

${ }^{22}$ Gaston Paris, De Pseudo-Turpino, Paris, Franck, 1865, p. 57.

${ }^{23}$ Ronald N. Walpole, The Old French Johannes Translation..., op. cit., pp. 83-96.

${ }^{24}$ Ibidem, p. 95.

${ }^{25}$ Ian Short, The Anglo-Norman Pseudo-Turpin..., op. cit., p. 2.

${ }^{26}$ Gabrielle M. Spiegel, «Pseudo-Turpin, the crisis of the aristocracy and the beginnings of the vernacular historiography in France», Journal of Medieval History, 12-3 (1986), pp. 207223, spec. p. 210. DOI: https://doi.org/10.1016/0304-4181(86)90032-1.

${ }^{27}$ Come osservò Ian Short (The Anglo-Norman Pseudo-Turpin..., op. cit., p. 7), le prime attestazioni della presenza del cappellano presso la corte di Re Giovanni datano al 1212-13.

${ }^{28}$ Marco Piccat, Lo Pseudo-Turpino..., op. cit., pp. 30-34. 
Sul piano editoriale, ad oggi risulta pubblicato dunque solo il secondo rimaneggiamento della 'Johannes', mentre manca l'edizione critica delle altre due versioni: della traduzione originale (famiglia 1) esistono infatti soltanto due edizioni ${ }^{29}$, ormai assai datate e non critiche in quanto fondate ciascuna su di un solo manoscritto; del tutto inedito è poi il primo rifacimento (famiglia 2 ).

La mole degli studi di Walpole ha senza dubbio esercitato un effetto inibitorio sull'implementazione delle ricerche: dopo un periodo di 'esplosione', che culminò grosso modo con gli anni ' 70 del secolo scorso, il ritmo pare rallentare notevolmente, come se tutto fosse ormai stato appurato e nulla di nuovo potesse essere aggiunto. In realtà, margini di significativo progresso esistono ancora.

L'equivoco ingenerato dal titolo dell'edizione Walpole non costituisce in effetti l'unico problema: a ben guardare, la traditio appare tratteggiata nelle grandi linee ma risulta ancora imperfetta su scala minore, con filiazioni e contaminazioni solo parzialmente definite; anche il censimento e la recensio dei codici suscita più di una perplessità, sia riguardo alla completezza - da quanto Walpole scrive ${ }^{30}$, sorge infatti il dubbio che essa possa dirsi conclusa: molti sono infatti, per sua stessa ammissione, i manoscritti di compilazioni storiche che potrebbero veicolare il testo, sui quali egli non riuscì a condurre una ricerca sistematica - che, soprattutto, dal punto di vista dei suoi risultati, tradotti nelle ricostruzioni stemmatiche.

Ad esempio Walpole annovera nella seconda famiglia, inserendolo nel relativo stemma, un manoscritto (Modena, Estense N.5.12, sigla: Mo) che non contiene né ha mai contenuto la Cronaca dello Pseudo-Turpino:

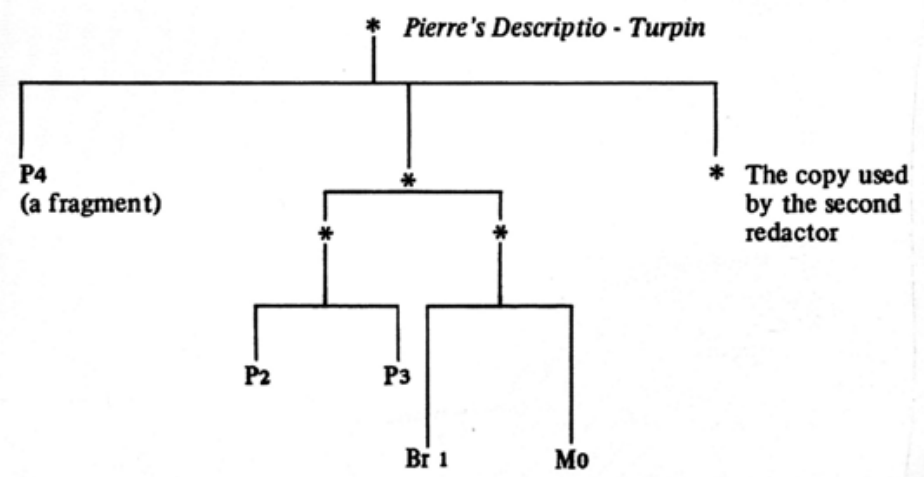

${ }^{29}$ Theodor Auracher, «Der Altfranzösische Pseudo-Turpin der Arsenalhandschrift BLF 283», Romanische Forschungen, V (1889), pp. 137-171; Rudolf Schmitt, Der Pseudo-Turpin Harley 273. Der text mit eine Untersuchung der Sprache, Würzburg, Romanischen Seminars der Universitat Wurzburg, 1933.

${ }^{30}$ Ronald N. Walpole, The Old French Johannes Translation..., op. cit., p. XX. 
Il copista di Mo, è interessato, come lo stesso Walpole dichiara ${ }^{31}$, solo alla Descriptio, in quanto in linea sul piano contenutistico con gli altri testi del codice miscellaneo; in conseguenza di ciò, egli non solo non copia la Cronaca, ma addirittura elimina sistematicamente ogni riferimento al Turpino nel prologo della Descriptio. Se così è, non si comprende come tale codice abbia titolo a comparire nello stemma insieme agli altri che recano Descriptio e Cronaca.

Nello stemma inoltre, dopo aver individuato la parentela fra i codici P2 (Paris, BnF, fr. 834) e P3 (Paris, BnF, nouv acq. fr. 13521), Walpole afferma che $\langle\mathrm{P} 2 \text { is slightly the better manuscript }\rangle^{32}$, qualità migliore che tuttavia non appare dal grafo, in cui i due codici sono posti esattamente sullo stesso piano.

Ancor più sorprendente appare lo scollamento fra stemma e scelte editoriali riguardo alla terza famiglia della 'Johannes' 33 , e ciò ancor più se si considera che l'edizione Walpole verte proprio su questa versione:

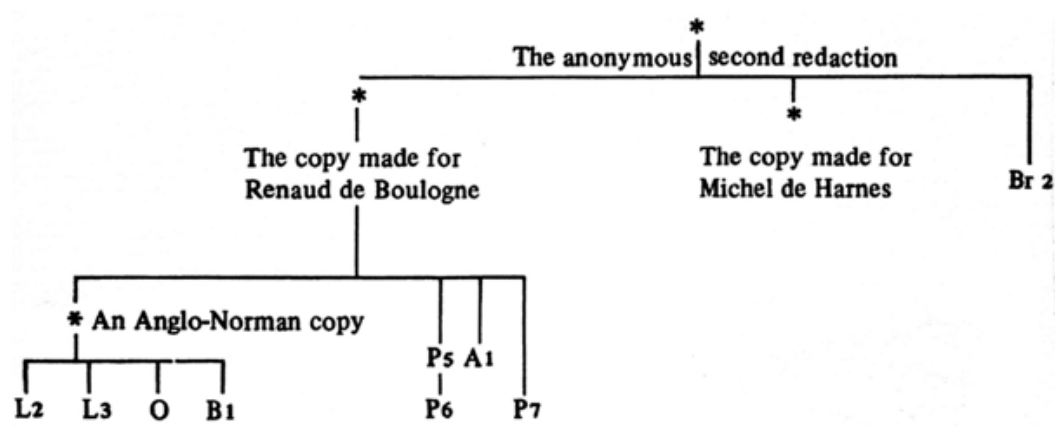

Lo studioso individua il manoscritto base in P5 (Paris, BnF, fr. 2464), contrariamente a quanto evidenziato nello stemma, in cui la posizione di codice più vicino all'archetipo è occupata da L2 (London, BM, Royal MS 4 C. xi); i manoscritti di controllo vengono indicati nella sequenza $\mathrm{A}_{1}$ (Paris, Arsenal, 5201), $\mathrm{L}_{2}, \mathrm{P}_{8}$ (Paris, $\mathrm{BnF}$, fr. 1444), V (Roma, BAV, Regina 936), $\mathrm{Br}_{2}$ (Bruxelles, KBR, 1023336) ${ }^{34}$; ora, $\mathrm{P}_{8}$ e V appartengono al secondo sottogruppo; in fase di emendatio, si registrerebbe così uno strano andamento ondivago fra $\mathrm{i}$ diversi rami della tradizione; del tutto improponibile risulta poi l'ipotesi (p. 126) di usare $\mathrm{P}_{6}$ (Paris, $\mathrm{BnF}$, nouv acq. fr. 10554), copia di $\mathrm{P}_{5}$, per emendare il manoscritto base: se è un descriptus, non può servire a questo scopo se non limitatamente a eventuali guasti meccanici di $\mathrm{P}_{5}$, ma se fosse davvero un descriptus, non dovrebbe presentare lezioni corrette là dove $\mathrm{P}_{5}$ sbaglia, cosa che invece accade, come

\footnotetext{
${ }^{31}$ Ibidem, p. 27.

${ }^{32}$ Ibidem, p. 46.

${ }^{33}$ Ibidem, p. 66.

${ }^{34}$ Ibidem, p. 125.
} 
l'editore (p. 62) dimostra. Walpole giustifica il fatto con l'abilità del copista di $\mathrm{P}_{6}$ nell'identificare e correggere le eventuali lezioni erronee, attribuendogli capacità francamente sovrastimate, in quanto il suo giudizio risulta a mio avviso viziato da due errori metodologici di base: in primo luogo la dipendenza di P6 da P5 non viene provata correttamente: l'uguale sequenza di testi all'interno dei due manoscritti e la convergenza di P5 e P6 su alcune varianti, aggiunte e omissioni non sono dati sufficienti a dimostrare che l'uno è copia dell'altro; la prova inconfutabile che porterebbe a questa conclusione, e cioè la presenza in P6 di tutti gli errori di P5, in aggiunta ad alcuni propri, manca; in secondo luogo l'ipotesi della dipendenza dei due codici da un antigrafo comune, che potrebbe agevolmente spiegare le lezioni buone di P6 senza dover scomodare le capacità emendative del suo copista, viene categoricamente esclusa da Walpole senza ulteriori argomentazioni: "The question arises here as to whether these readings which I attribute as emendations to the scribe of P6 as he copied P5, are of such a nature as to suggest rather that P5 and P6 are copied from a common source. I do not think so» (p. 62).

$\mathrm{Da}$ ultimo, lo stemma non registra lo scarto temporale (più di 200 $a_{n n i}{ }^{35}$ ) che intercorre fra i manoscritti più antichi e i recentiores: nel grafo, che non rende la reale struttura della tradizione né in prospettiva cronologica, né filologica, i codici vengono posti sostanzialmente sullo stesso piano.

Egli lascia poi in sospeso la cruciale questione dei prologhi nei codici della terza famiglia: il loro contenuto alimenta il dubbio che tale gruppo annoveri in realtà due traduzioni, una rinvenuta da Renaud de Boulogne a Saint-Denis e l'altra tratta da Michel de Harnes dalla biblioteca dello stesso Renaud. L'editore tuttavia soprassiede sul problema, rimandando ogni soluzione alla collazione dei manoscritti: «But this particular problem must wait, for we can see that its solution will depend in great part on a thorough collation of the manuscripts $»^{36}$.

Questi livelli di approssimazione non riguardano d'altra parte la sola versione 'Johannes'; analoghe incongruenze si registrano anche in relazione alle altre traduzioni di cui Walpole si occupò: per la seconda versione anonima (Turpin II), egli elabora uno stemma che appare in netto contrasto con le sue affermazioni:

${ }^{35} \mathrm{~L}_{2}$ : 1225; $\mathrm{L}_{3}$ (London, BM, add. 40142): 1250 ca.; O (Oxford, Bodleian Library, Hatton 67), $\mathrm{B}_{1}$ (Bern, Bürgerbibliothek, MS 115), $\mathrm{A}_{1}$ : seconda metà XIII sec.; $\mathrm{P}_{5}$ : 1225-1250 ca.; $\mathrm{P}_{6}$ : 1450-1475 ca.; $\mathrm{P}_{7}$ (Paris, BnF, fr. 5713): 1450 ca.

${ }^{36}$ Ronald N. Walpole, The Old French Johannes Translation..., op. cit., p. 12. 


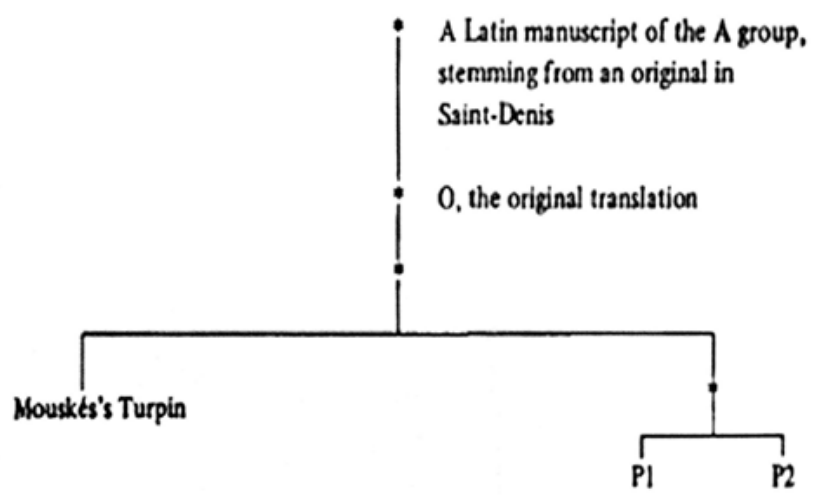

Walpole ${ }^{37}$ sostiene che P2 (Paris, BnF, fr. 17203) presenti lezioni migliori di P1 (Paris, BnF, fr. 2137), ma nello stemma colloca i due codici allo stesso livello; in secondo luogo egli afferma che la versione utilizzata da Mouskés è più vicina a $\mathrm{P} 2$ che a $\mathrm{P} 1^{38}$, ma la struttura del grafo mostra l'esatto contrario; ancora una volta inoltre i rapporti di vicinanza con l'archetipo non paiono correttamente rappresentati, in quanto dallo stemma si dedurrebbe che sia la versione di Mouskés a veicolarne il testo; in sostanza il testimone migliore (P2) è quello che viene collocato in posizione più lontana.

Le stesse problematiche ricompaiono in relazione alla prima versione anonima (Turpin I): l'edizione Walpole è basata sul manoscritto siglato $\mathrm{A}(\text { Paris, } \mathrm{BnF} \text {, fr. } 1850)^{39}$, il migliore e più antico; nell'albero genealogico ${ }^{40}$ il codice è invece collocato lontano dall'archetipo e in posizione più bassa rispetto ad altri testimoni più tardi; $\mathrm{B}$ (Roma, BAV, Regina 624) e C (Chantilly, Musée Condé, MS 869), vergati a duecento di distanza l'uno dall'altro, sono posti sullo stesso piano, mentre G (Firenze, Laurenziana, Ashburnham 54), coevo di C, è collocato molto più in basso rispetto ad esso. È pur vero che districare $\mathrm{i}$ rapporti fra i testimoni di questa versione non è affare di poco conto, ma i difetti stemmatici ora evidenziati prescindono da questo aspetto.

Che si tratti di un volgarizzamento ad alto tasso di problematicità è d'altra parte dimostrato dalle sue vicende editoriali: nel 1976 Claude Buridant ${ }^{41}$ pubblicò il testo della Cronaca contenuto nel ms. Vat. Regina 624, nella convinzione che si trattasse di una traduzione

${ }^{37}$ Ronald N. Walpole, An Anonymous Old French Translation..., op. cit., p. 27.

${ }^{38}$ Ibidem, p. 26.

${ }^{39}$ Per la descrizione dei manoscritti cfr. Ronald $\mathrm{N}$. Walpole, «Prolégomènes à une édition du Turpin français dit Turpin I», Revue d'histoire des textes, 10 (1980), pp. 199-230 [Online]. Link: <http://www.persee.fr/doc/rht_0373-6075_1982_num_10_1980_1219> [Consultato: 18/01/2018). DOI: 10.3406/rht.1982.1219.

${ }^{40}$ Ronald N. Walpole, Le Turpin français, dit le Turpin I..., op. cit., p. XIX.

${ }^{41}$ Claude Buridant, La traduction du Pseudo-Turpin du manuscrit Vatican Regina 624, édition avec introduction, notes et glossaire, Genève, Droz, 1976. 
indipendente; di lì a poco Walpole ${ }^{42}$ recensì negativamente tale edizione ribadendo la sua teoria in base alla quale il codice, siglato B, non sarebbe che uno dei testimoni della prima versione anonima; l'errore cruciale di Buridant fu quello di considerare l'indipendenza di B come un assioma, mentre si tratterebbe se mai di un teorema in attesa di dimostrazione; egli confrontò il testo, limitatamente ai piani dello stile e delle scelte traduttive, in primis con quello dell'ed. Short e in seconda istanza con le carenti edizioni di Wulff e di Fischer ${ }^{43}$ allora disponibili; una recensio completa, seguita da un rigoroso esame filologico, avrebbe forse fornito qualche elemento in più a favore della sua tesi, anche perché la controanalisi condotta da Walpole non convince su molti punti: consapevole del fatto che «l'établissement d'un texte critique du Turpin I présente des problèmes complexes et ardus ${ }^{44}$, egli si sforza di evidenziare le convergenze di B con gli altri testimoni, pur andando in crisi di fronte a «des variantes qui viennent obscurcir un peu les éléments de nos démonstrations» (p. 503) e gettare definitivamente la spugna quando la deriva di B pare essere senza più controllo: «inutile donc ici de faire entrer le texte de $\mathrm{B}$ dans notre collation. Il traite son modèle avec une très grande, pour ne pas dire une énorme, liberté» (p. 504). Di fronte a una situazione di questo tipo, la temporanea sospensione del giudizio è d'obbligo; resta il fatto che la posizione di Walpole ne condiziona l'edizione: egli la fonda sul ms. A, ponendo in apparato le varianti di C, D (Paris, BnF, nouv. acq. fr. 6295), E (Roma, BAV, Regina lat. 610), ma solo episodicamente quelle di B («j'ai recours... aux textes de C D E, parfois à celui de $\mathrm{B} »)^{45}$, cioè solo quando esse sembrano dipendere dall'antigrafo «et non pas du caprice du scribe»; non solo, ma nonostante D e E abbandonino il testo della prima versione anonima dal cap. XIV per riprodurre la versione 'Johannes', egli continua inspiegabilmente a riportare le loro varianti.

\section{CODICI DELLA 'JOHANNES': UNA PRIMA RICOGNIZIONE}

Sulla base di queste considerazioni, appare del tutto giustificato un riesame della tradizione manoscritta dell'originaria versione 'Johannes' in chiave propedeutica all'allestimento dell'edizione critica tuttora mancante; Walpole ${ }^{46}$ elencò quattro codici - ms. Paris, BnF, fr. 1621, siglato P1, ms. London, British Library, Harley 273, siglato L1, ms. Munich, Bayerische Staadstbibliotheck, Gallicus 52, siglato M1, e il deperditus ms. Dover, St. Martin's Priory, H. VII. 6,

\footnotetext{
${ }^{42}$ Ronald N. Walpole, «La traduction du Pseudo-Turpin du manuscrit Vatican Regina 264. À propos d'un livre récent», Romania, 99 (1978), pp. 484-514.

${ }^{43}$ Pius P. Fischer, Die französische Uebersetzung des Pseudo-Turpin nach dem Codex Gallicus 52 (München), Wertheim-am-Main, E. Berchstein Inh. W. Hinckel, 1932.

${ }^{44}$ Ronald N. Walpole, «La traduction du Pseudo-Turpin...», art. cit., p. 494.

${ }^{45}$ Ronald N. Walpole, Le Turpin français, dit le Turpin I..., op. cit., p. XXI.

${ }^{46}$ Ronald N. Walpole, The Old French Johannes Translation..., op. cit., p. 2.
} 
siglato D - elaborando anche uno stemma articolato in tre famiglie, alla prima delle quali apparterrebbero M e P1.

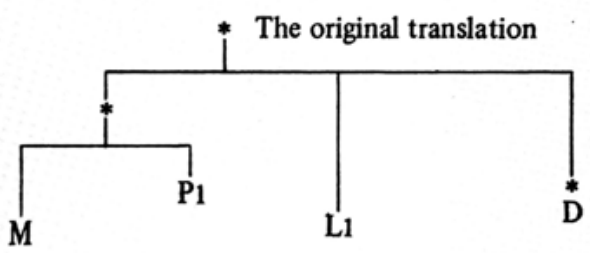

Da un primo confronto pare tuttavia evidenziarsi una situazione differente, in quanto P1 sembra offrire lezioni diverse rispetto agli altri codici, e ciò sin dal prologo; la versione 'Johannes' originaria è contraddistinta da un incipit

Chi commenche l'estoire que Torpins li archevesques de Rains fist, et traita du bon roi Charlemaine, comment il conquist Espaigne et delivra des mains as paiens, et por che que l'estoire traitié par rime samble menchonge, est cheste sans rime mise en romans selonc le raison del latin. (P1, c. 238r.)

che compare, con talune varianti, nei tre testimoni; tuttavia, mentre in P1 il proemio si conclude con questa frase:

Huimais commencherai par quel raison et par quel achoison Charlesmaine s'esmut por aler en Espaigne, et Torpins avoc lui, qui tot metoit en escrit les choses qui au roi et a chaus de l'ost avenoient, et por che qu'il fust as vivans et as haus homes en mimoire et raconté qui a venir estoient et i presissent garde et lor sovenist des anchiseurs.

sia M1 (c. 102r.) che L1 (c. 86r.) presentano evidenti tagli:

M1: Huimais commencherai par quele ochoison et comment Charles vint en Espaigne et Turpins avoec lui, qui tout mist en escript pour chou que la verité fust aprés iaus en memore.

L1 Huimés comenceray coment e par qel enchesun Charlemaine vint en Espaigne e Turpin ou li, qi tut mist en escrit qe la verité fust aprés li en memoire.

M1 si caratterizza inoltre per un'altra particolarità, in quanto il testo che esso veicola esordisce con il prologo della fase tre della tradizione, sottofamiglia $b$

Voirs est que pluiseurs ont oï volentiers et oent encore de Charlemaine comment il conquist Espaigne et Galisce; mais quoique li autre i 
aient osté ne mis, ci poës oïr le verité d'Espaigne selonc le latin que Michiel de Harnes fist e par grant estude cerkier et querre es livres Renaut de Boulongne conte...

per poi interrompersi pressoché a metà e proseguire con il prologo recato da $\mathrm{P} 1$ e $\mathrm{L} 1$.

In altri casi è $\mathrm{P} 1$ a offrire lezioni più concise; ad esempio, al termine del cap. XIII che racconta come Agolant, intenzionato a ricevere il battesimo, cambi idea dopo aver visto come vengono trattati i poveri alla corte di Carlo, P1 (c. 212v.) così conclude:

Agolans repaira ariere a sa gent et lor conta la chose tele com il l'avoit veue et manda a Karlemaine bataille a l'endemain, et quant Charlemaine vit ce qu'il refuça bauptesme por les povres, s'en fu merveilles dolens; il les retint puis tote sa vie a table et a nape moult honorablement. Ichi devons nos garder que moult aquiert grant cope qui les povres Damledeu n'oneure. Se Karlemaine perdi .j. roi et se gent a bautisier por les povres, qu'iert il donc au deerrain jor de chaus qui les povres auront traitiés malement, quant Dex jugera le monde chas[c]un selon ses ovres?

mentre M1 (c. 106r.) e L1 (c. 92r.), con maggiore aderenza del primo alla fonte latina ${ }^{47}$, recano:

M1: Il s'en repaira a son ost et manda a Charle a l'endemain bataille; quant Charle vit qu'il refusa baptesme pour les povres, mout en fu dolans; il les rechut puis toute sa vie a table et a nape honnourablement. Chi devons nous prendre garde que grant coupe aquiert cil qui les povres Dieu ne honneure. Se Charle perdi un roi et sa gent a baptisier pour les povres, que sera dont de ceus au darrain jour qui les povres aront traitiés mauvaisement? Comment orront il la vois Diu qui dira: «Vous maudis, departés de moi! Alés ou maudit feu d'enfer, car j'ai eu froit, fain et soif et vous ne m'avés pas regardé!»». Chi devons nous entendre que la loy de Diu et sa foi vaut pau en crestiens sans bonnes oevres, car l'Escripture dist: si comme le cors est mors sans l'ame, aussi est en l'omme foy morte sans bonne oevre; si comme le paiien refusa le baptesme, si devons nous cremir que Dieu ne nous refuse au jour del jugement pour ce qu'il ne troeve mie en nous oevres droiturieres selonc le baptesme.

L1: ...e se repeira a sun ost e l'endemain manda bataille; quant Charle ceo oÿt, mut en fut dolent e honora les povres mult granment, car grant blame se atret qe les povres deshonoure, e au jur de juyse Deu dirra as malorees: «Descendét en pardurable peine, car quant

${ }^{47}$ Cfr. Cyril Meredith-Jones (ed.), Historia Karoli Magni et Rotholandi ou Chronique du Pseudo-Turpin, Paris, Droz, 1936, pp. 138-139. 
jeo avoy feim, seif e freid vus ne me regardastes!», car creistienetee valt poi sanz bones eoveres; si cum le paen refusa baptesme, ausi devun entendre qe Deu se coroucera vers nus si il ne treove mie en nus dreites eoveres de baptesme.

Questi pochi esempi ${ }^{48}$, insieme al quadro generale degli studi prima delineato, dimostrano l'urgenza di una ricomposizione del mosaico delle versioni turpiniane oitaniche; date le problematiche ora evidenziate, l'edizione della 'Johannes' originaria ora in preparazione potrà costituire la prima tessera nel quadro di un auspicabile rinnovato interesse per le versioni galloromanze dello Pseudo Turpino.

Ricevuto: 5/02/2018

Accettato: 21/10/2018

${ }^{48}$ L'ultimo caso illustrato parrebbe evidenziare un'affinità di P1 con il codice latino B3 (Paris, BnF, lat. 5452), che presenta la stessa conclusione abbreviata del capitolo (cfr. Ibidem, p. 139); tale indizio andrà verificato in sede di un'esaustiva analisi testuale. 


\title{
$\cos$
}

\section{PER UN'EDIZIONE DELLA VERSIONE 'JOHANNES' DELL'HISTORIA KAROLI MAGNI ET ROTHOLANDI}

\begin{abstract}
Riassunto: La tradizione testuale della Cronaca dello Pseudo Turpino in area galloromanza rivela una complessità solo in parte chiarita dagli studi esistenti. La traditio dei volgarizzamenti appare tratteggiata nelle grandi linee ma risulta ancora imperfetta su scala minore, con filiazioni e contaminazioni solo parzialmente definite; anche la recensio presenta vari problemi, sia riguardo alla sua completezza che, soprattutto, dal punto di vista delle ricostruzioni stemmatiche. La disamina di queste problematiche costituisce la fase preparatoria dello studio della versione 'Johannes', di cui a tutt'oggi manca l'edizione critica, obiettivo finale dell'indagine.
\end{abstract}

Parole chiave: Pseudo-Turpino, epica, Francia, questioni ecdotiche.

\section{THE 'JOHANNES' VERSION OF THE HISTORIA KAROLI MAGNI ET ROTHOLANDI: A PRELIMINARY STUDY}

\begin{abstract}
The textual tradition of the Pseudo Turpin Chronicle in the GalloRoman area reveals a complexity only partially clarified by the existing studies. The traditio of the French vernacular versions appears dashed in the main lines but is still imperfect on a smaller scale, with only partially defined filiations and contaminations; also the recensio presents various problems, both regarding its completeness and, above all, from the point of view of the stemmatic reconstructions. The analysis of these problems constitutes the preparatory phase of the study of the 'Johannes' version, which is still missing the critical edition, the final objective of this research.
\end{abstract}

KeYwords: Pseudo-Turpin, Epic, France, ecdotic problems. 